Bull. Korean Math. Soc. 52 (2015), No. 2, pp. 525-530

http://dx.doi.org/10.4134/BKMS.2015.52.2.525

\title{
UPPERS TO ZERO IN POLYNOMIAL RINGS WHICH ARE MAXIMAL IDEALS
}

\author{
Gyu Whan Chang
}

\begin{abstract}
Let $D$ be an integrally closed domain with quotient field $K$, $X$ be an indeterminate over $D, f=a_{0}+a_{1} X+\cdots+a_{n} X^{n} \in D[X]$ be irreducible in $K[X]$, and $Q_{f}=f K[X] \cap D[X]$. In this paper, we show that $Q_{f}$ is a maximal ideal of $D[X]$ if and only if $\left(\frac{a_{1}}{a_{0}}, \ldots, \frac{a_{n}}{a_{0}}\right) \subseteq P$ for all nonzero prime ideals $P$ of $D$; in this case, $Q_{f}=\frac{1}{a_{0}} f D[X]$. As a corollary, we have that if $D$ is a Krull domain, then $D$ has infinitely many heightone prime ideals if and only if each maximal ideal of $D[X]$ has height $\geq 2$.
\end{abstract}

\section{Introduction}

Let $D$ be an integral domain with quotient field $K, \bar{D}$ the integral closure of $D$ in $K, X$ an indeterminate over $D$, and $D[X]$ the polynomial ring over $D$. We say that a nonzero prime ideal $Q$ of $D[X]$ is an upper to zero in $D[X]$ if $Q \cap D=(0)$; so each upper to zero in $D[X]$ has height-one. Clearly, if $Q$ is an upper to zero in $D[X]$, then $Q=f K[X] \cap D[X]$ for some $f \in D[X]$ which is irreducible in $K[X]$.

Let $X_{1}, \ldots, X_{n}$ be indeterminates over $D$. It is known that the intersection of the nonzero prime ideals of $D$ is zero if and only if $M \cap D \neq(0)$ for all maximal ideals $M$ of $D\left[X_{1}, \ldots, X_{n}\right]$ [11, Theorem 14.10]. This result was first proved by Artin-Tate [2] in the Noetherian case and then by Nagata [11] in the general case. In [10], May used this result to give an elementary proof of the Nullstellensatz: if $F$ is an algebraically closed field, then an ideal $M$ of $F\left[X_{1}, \ldots, X_{n}\right]$ is maximal if and only if $M=\left(X_{1}-a_{1}, \ldots, X_{n}-a_{n}\right)$ for $a_{1}, \ldots, a_{n} \in F$. Let $A$ be the intersection of the nonzero prime ideals of $D$. It is clear by [11, Theorem 14.10] that $A \neq(0)$ if and only if there is a maximal ideal $M$ of $D[X]$ with $M \cap D=(0)$. In this case, if we let $f=1+a X$ for $0 \neq a \in A$, then $Q_{f}:=f K[X] \cap D[X]$ is a maximal ideal of $D[X]$ with $Q_{f} \cap D=(0)$ [9, Proof of Theorem 24] and $Q_{f}=f D[X]$. In this paper, we completely characterize uppers to zero in $D[X]$ that are maximal ideals.

Received February 21, 2014.

2010 Mathematics Subject Classification. 13A15, 13B25, 13G05.

Key words and phrases. upper to zero, maximal ideal, polynomial ring, G-domain. 
Let $f=a_{0}+a_{1} X+\cdots+a_{n} X^{n} \in D[X]$ be such that $f$ is irreducible in $K[X]$ and $Q_{f}=f K[X] \cap D[X]$. We show that $\sqrt{f D[X]}$ is a maximal ideal of $D[X]$ if and only if $a_{0}$ is a unit in $D$ and $\left(a_{1}, \ldots, a_{n}\right) \subseteq P$ for all nonzero prime ideals $P$ of $D$. Then we use this result to show that if $D$ is integrally closed, then $Q_{f}$ is a maximal ideal if and only if $\left(\frac{a_{1}}{a_{0}}, \ldots, \frac{a_{n}}{a_{0}}\right) \subseteq P$ for all nonzero prime ideals $P$ of $D$; in this case, $Q_{f}=\frac{1}{a_{0}} f D[X]$. Also, we give an example of a non-integrally closed integral domain $D$ with $g \in D[X]$ such that $g K[X] \cap D[X]$ is a maximal ideal, but not a principal ideal. Finally, we show that if $D$ is a Krull domain, then $D$ has infinitely many height-one prime ideals if and only if each maximal ideal of $D[X]$ has height $\geq 2$.

Let $I$ be a nonzero fractional ideal of $D$. Then $I^{-1}=\{x \in K \mid x I \subseteq D\}$, $I_{v}=\left(I^{-1}\right)^{-1}$, and $I_{t}=\cup\left\{J_{v} \mid J \subseteq I\right.$ is a nonzero finitely generated ideal $\}$. We say that $I$ is a $t$-ideal if $I_{t}=I$. A $t$-ideal of $D$ is a maximal $t$-ideal if it is maximal among proper integral $t$-ideals. Let $t$-Max $(D)$ denote the set of maximal $t$-ideals of $D$. It is well known that a maximal $t$-ideal is a prime ideal; each integral $t$-ideal is contained in a maximal $t$-ideal; $D=\cap_{P \in t \text {-Max }(D)} D_{P}$; and each prime ideal minimal over a $t$-ideal is a $t$-ideal (and hence each heightone prime ideal is a $t$-ideal and $t$ - $\operatorname{Max}(D) \neq \emptyset$ if $D$ is not a field). A nonzero ideal $I$ of $D$ is said to be $t$-invertible if $\left(I I^{-1}\right)_{t}=D$; equivalently, $I I^{-1} \nsubseteq P$ for all $P \in t-\operatorname{Max}(D)$.

\section{Main results}

Let $D$ be an integral domain with quotient field $K, X$ be an indeterminate over $D$, and $D[X]$ be the polynomial ring over $D$.

Lemma 1. Let $f=a_{0}+a_{1} X+\cdots+a_{n} X^{n} \in D[X]$ and $I$ an ideal of $D$. Then $f D[X]+I[X]=D[X]$ if and only if $a_{0} D+I=D$ and $\left(a_{1}, \ldots, a_{n}\right)^{m} \subseteq I$ for some integer $m \geq 1$.

Proof. Note that $f D[X]+I[X]=D[X]$ if and only if $D[X] / I[X] \cong(D / I)[X]=$ $(\bar{f}) ; a_{0} D+I=D$ if and only if $\overline{a_{0}}$ is a unit in $D / I$; and $\overline{a_{1}}, \ldots, \overline{a_{n}}$ are nilpotent in $D / I$ if and only if $a_{i}^{m_{i}} \in I$ for some integer $m_{i} \geq 1$, if and only if $\left(a_{1}, \ldots, a_{n}\right)^{m} \subseteq I$ for some integer $m \geq 1$. Thus the result follows directly from [3, Exercise 2, page 10] that $g=b_{0}+b_{1} X+\cdots+b_{m} X^{m} \in(D / I)[X]$ is a unit if and only if $b_{0}$ is a unit in $D / I$ and $b_{1}, \ldots, b_{m}$ are nilpotent in $D / I$.

Let $f=a_{0}+a_{1} X+\cdots+a_{n} X^{n} \in D[X]$. We denote by $c_{D}(f)$ (or simply $c(f)$ ) the ideal of $D$ generated by the coefficients of $f$, i.e., $c_{D}(f)=\left(a_{0}, a_{1}, \ldots, a_{n}\right)$. It is well known that if $g, h \in D[X]$, then there exists a positive integer $m$ such that $c(g)^{m+1} c(h)=c(g)^{m} c(g h)$ [5, Theorem 28.1]. Using this result, we can easily show that $f K[X] \cap D[X]=f D[X]$ if and only if $c(f)^{-1}=D$ (see, for example, [1, Lemma 2.1(1)]). Also, it is well known that if $D$ is integrally closed, then $g K[X] \cap D[X]=g c(g)^{-1}[X]$ for all $0 \neq g \in D[X][5$, Corollary $34.9]$. 
Lemma 2. Let $f=a_{0}+a_{1} X+\cdots+a_{n} X^{n} \in D[X]$ be irreducible in $K[X]$. Then $\sqrt{f D[X]}$ is a maximal ideal of $D[X]$ if and only if $a_{0}$ is a unit in $D$ and $\left(a_{1}, \ldots, a_{n}\right) \subseteq P$ for all nonzero prime ideals $P$ of $D$. In this case, $\sqrt{f D[X]}=$ $f D[X]$.

Proof. $(\Rightarrow)$ Suppose that $\sqrt{f D[X]}$ is a maximal ideal of $D[X]$, and let $P$ be a nonzero prime ideal of $D$; then $P \nsubseteq \sqrt{f D[X]}$, and hence $f D[X]+P[X]=D[X]$. Thus by Lemma $1, a_{0} \notin P$ and $\left(a_{1}, \ldots, a_{n}\right) \subseteq P$. In particular, since $P$ is arbitrary, $a_{0}$ must be a unit in $D$.

$(\Leftarrow)$ First, note that $f K[X] \cap D[X]=f D[X]$ since $a_{0}$ is a unit in $D$. Let $Q$ be a prime ideal of $D[X]$ with $f \in Q$. If $Q \cap D \neq(0)$, then $\left(a_{1}, \ldots, a_{n}\right) \subseteq Q \cap D$ and $f D[X]+(Q \cap D)[X] \subseteq Q$. Note that $a_{0} D+Q \cap D=D$ because $a_{0}$ is a unit; so by Lemma $1, D[X]=f D[X]+(Q \cap D)[X] \subseteq Q$, a contradiction. Hence $Q \cap D=(0)$, and thus $Q=f K[X] \cap D[X]$ and $Q$ is the unique prime ideal of $D[X]$ that contains $f$. Therefore $\sqrt{f D[X]}=Q=f D[X]$ is a maximal ideal.

We are now ready to prove the main result of this paper.

Theorem 3. Let $D$ be an integrally closed domain, $f=a_{0}+a_{1} X+\cdots+a_{n} X^{n} \in$ $D[X]$ be such that $f$ is irreducible in $K[X]$, and $Q_{f}=f K[X] \cap D[X]$. Then $Q_{f}$ is a maximal ideal of $D[X]$ if and only if $\left(\frac{a_{1}}{a_{0}}, \ldots, \frac{a_{n}}{a_{0}}\right) \subseteq P$ for all nonzero prime ideals $P$ of $D$. In this case, $Q_{f}=\frac{1}{a_{0}} f D[X]$.

Proof. $(\Rightarrow)$ Since $Q_{f}$ is maximal, $Q_{f}$ is a maximal $t$-ideal, and hence $Q_{f}$ is $t$ invertible [7, Theorem 1.4]. Note that $Q_{f}=f c_{D}(f)^{-1}[X]$ and $(I D[X])_{t}=$ $I_{t} D[X]$ for all nonzero fractional ideals $I$ of $D$ [6, Proposition 3.4]; hence $c_{D}(f)^{-1}$ is $t$-invertible. Let $P$ be a maximal $t$-ideal of $D$. Then $\left(Q_{f}\right)_{D \backslash P}$ is a maximal ideal of $D_{P}[X]$. Note that $c_{D}(f) c_{D}(f)^{-1} \nsubseteq P$; so $c_{D}(f) D_{P}$ is invertible. Hence $c_{D}(f) D_{P}=\left(c_{D}(f) D_{P}\right)_{t}=\left(c_{D}(f)_{t} D_{P}\right)_{t} \supseteq c_{D}(f)_{t} D_{P} \supseteq c_{D}(f) D_{P}$ [8, Lemma 3.4(3)] and $c_{D}(f) D_{P}=a_{i} D_{P}$ for some $i$ [5, Proposition 7.4(2)], and thus $c_{D}(f)_{t} D_{P}=a_{i} D_{P}$. Note also that $\left(Q_{f}\right)_{D \backslash P}=f K[X] \cap D_{P}[X]=$ $\frac{1}{a_{i}} f D_{P}[X]$. Hence by Lemma $2, \frac{a_{0}}{a_{i}}$ is a unit in $D_{P}$; so $a_{i} D_{P}=a_{0} D_{P}$. Thus $c_{D}(f)_{t}=\cap_{P \in t-\operatorname{Max}(D)} c_{D}(f)_{t} D_{P}=\cap_{P \in t-\operatorname{Max}(D)} a_{0} D_{P}=a_{0} D$ [8, Proposition $2.8(3)]$. This implies that $Q_{f}=f K[X] \cap D[X]=\frac{1}{a_{0}} f D[X]$. Again, by Lemma $2,\left(\frac{a_{1}}{a_{0}}, \ldots, \frac{a_{n}}{a_{0}}\right) \subseteq P$ for all nonzero prime ideals $P$ of $D$.

$(\Leftarrow)$ Let $h=\frac{1}{a_{0}} f=1+\frac{a_{1}}{a_{0}} X+\cdots+\frac{a_{n}}{a_{0}} X^{n}$. Then by Lemma $2, Q_{f}=$ $h K[X] \cap D[X]=h D[X]$ is a maximal ideal of $D[X]$.

We next give an example which shows that Theorem 3 does not hold for a non-integrally closed domain.

Example 4. Let $D$ be a one-dimensional quasi-local domain with maximal ideal $P$ such that $\bar{D}$ is quasi-local with maximal ideal $Q$ and $P \bar{D} \subsetneq Q$. (For example, let $F$ be a field, $t$ an indeterminate over $F$, and $D=F \llbracket t^{2}, t^{3} \rrbracket$ be a subring of the power series ring $F \llbracket t \rrbracket$. Then $\left(t^{2}, t^{3}\right)$ is a maximal ideal of 
$D, \bar{D}=F \llbracket t \rrbracket$ is a local PID with maximal ideal $t \bar{D}$, and $\left(t^{2}, t^{3}\right) \bar{D} \subsetneq t \bar{D}$.) Choose $u=\frac{b}{a} \in Q \backslash P \bar{D}$, where $a, b \in D$, and let $f=a+b X \in D[X]$. Then $f K[X] \cap D[X]$ is a maximal ideal of $D[X]$, but not a principal ideal.

Proof. Obviously, $f K[X] \cap \bar{D}[X]=f c_{\bar{D}}(f)^{-1}[X]=\frac{1}{a} f \bar{D}[X]$. Hence $f K[X] \cap$ $\bar{D}[X]$ is a maximal ideal by Theorem 3 , and thus $f K[X] \cap D[X]$ is a maximal ideal of $D[X]$ because $\bar{D}[X]$ is integral over $D[X]$. Next, assume that $f K[X] \cap$ $D[X]$ is principal. Then there exists an $h \in D[X]$ such that $f K[X] \cap D[X]=$ $h D[X]$. Note that $h K[X]=f K[X]$; so $f=\alpha h$ for some $\alpha \in K$ and $c_{D}(h)=D$. Hence $(a, b)=c_{D}(f)=\alpha D$, and thus $(a, b)$ is invertible. But, in this case, either $(a, b)=a D$ or $(a, b)=b D$ because $D$ is quasi-local. If $(a, b)=a D$, then $\frac{b}{a} \in D \subseteq \bar{D}$, and hence $\frac{b}{a} \in Q \cap D=P \subseteq P \bar{D}$, a contradiction. Assume $(a, b)=b D$. Then $\frac{a}{b} \in D \subseteq \bar{D}$, and hence $1=\frac{b}{a} \cdot \frac{a}{b} \in Q$, a contradiction. Thus $f K[X] \cap D[X]$ is not principal.

Corollary 5. Let $f=a_{0}+a_{1} X+\cdots+a_{n} X^{n} \in D[X]$ be such that $f$ is irreducible in $K[X]$. Then the following statements are equivalent.

(1) $Q_{f}:=f K[X] \cap D[X]$ is a maximal ideal of $D[X]$.

(2) $M_{f}:=f K[X] \cap \bar{D}[X]$ is a maximal ideal of $\bar{D}[X]$.

(3) $\left(\frac{a_{1}}{a_{0}}, \ldots, \frac{a_{n}}{a_{0}}\right) \subseteq P$ for all nonzero prime ideals $P$ of $\bar{D}$.

In particular, if $a_{0}$ is a unit in $D$, then $Q_{f}$ is maximal if and only if $\left(a_{1}, \ldots, a_{n}\right)$ $\subseteq P$ for all nonzero prime ideals $P$ of $D$.

Proof. (1) $\Leftrightarrow(2)$ This follows from [9, Theorem 44] because $M_{f} \cap D[X]=Q_{f}$ and $\bar{D}[X]$ is integral over $D[X]$. (2) $\Leftrightarrow(3)$ Theorem 3. The "in particular" part follows because $\bar{D}$ is integral over $D$.

Corollary 6. Let $R$ be a subring of $K$ containing $D, f \in D[X]$ be irreducible in $K[X]$, and $Q_{f}=f K[X] \cap D[X]$. If $Q_{f}$ is a maximal ideal, then $f K[X] \cap R[X]$ is a maximal ideal of $R[X]$.

Proof. Let $\bar{R}$ be the integral closure of $R$ in $K$. Then $\bar{D} \subseteq \bar{R} \subseteq K$, and hence each $a \in \bar{D}$ that is contained in all nonzero prime ideals of $\bar{D}$ is contained in all nonzero prime ideals of $\bar{R}$. Thus, by Corollary $5, f K[X] \cap R[X]$ is a maximal ideal of $R[X]$.

An integral domain $D$ is called a $G$-domain if $K=D\left[\frac{1}{c}\right]$ for some $0 \neq c \in D$. It is clear that $D$ is a G-domain if and only if the intersection of the nonzero prime ideals of $D$ is nonzero [4, Lemma 3].

Corollary 7. Let $A$ be the intersection of the nonzero prime ideals of $D$.

(1) $\left(\left[9\right.\right.$, Proof of Theorem 24]) If $f=1+a X$ for $0 \neq a \in A$, then $Q_{f}=$ $f K[X] \cap D[X]$ is a maximal ideal of $D[X]$ and $Q_{f}=f D[X]$.

(2) $([9$, Theorem 24]) $D$ is a G-domain (i.e., $A \neq(0))$ if and only if there exists a maximal ideal $M$ of $D[X]$ which satisfies $M \cap D=(0)$.

(3) (cf. [11, Theorem 14.10]) $A=(0)$ if and only if $M \cap D \neq(0)$ for all maximal ideals $M$ of $D[X]$. 
Proof. Note that $A \subseteq P$ for all nonzero prime ideals $P$ of $\bar{D}$; so if we let $B$ be the intersection of the nonzero prime ideals of $\bar{D}$, then $A \neq(0)$ if and only if $B \neq(0)$.

(1) It is clear that $f$ is irreducible in $K[X]$ and $Q_{f}=f D[X]$. Thus, the result is an immediate consequence of Corollary 5 .

(2) If $D$ is a G-domain, then $K=D\left[\frac{1}{c}\right]$ for some $0 \neq c \in D$. Clearly, $c \in A$. So if we set $f=1+c X$ and $Q_{f}=f K[X] \cap D[X]$, then $Q_{f}$ is a maximal ideal of $D[X]$ by (1) and $Q_{f} \cap D=(0)$. For the converse, note that $B \neq(0)$ by Corollary 5. Thus $A \neq(0)$.

(3) This follows from (2).

Let $X^{1}(D)$ be the set of height-one prime ideals of $D$. Clearly, if each nonzero prime ideal of $D$ contains a height-one prime ideal, then $\cap_{P \in X^{1}(D)} P$ is equal to the intersection of the nonzero prime ideals of $D$. So if $D$ is a Krull domain (resp., principal ideal domain (PID)), then the intersection of the nonzero prime ideals of $D$ is zero if and only if $\left|X^{1}(D)\right|=\infty$. Also, it is well known and easy to prove that $D$ is a Krull domain with $\left|X^{1}(D)\right|<\infty$ if and only if $D$ is a semilocal PID [4, Theorem 1]. Thus by Corollary $7(3)$, we have:

Corollary 8. A Krull domain D has infinitely many height-one prime ideals if and only if each maximal ideal of $D[X]$ has height $\geq 2$.

Corollary 9 ([12, Theorem 2]). A PID D has infinitely many non-associate prime elements if and only if each maximal ideal of $D[X]$ has height 2.

Proof. This follows because the (Krull) dimension of $D[X]$ over a PID $D$ is 2 .

We end this paper with a concrete example of uppers to zero in $D[X]$ that are maximal ideals. This also shows that the converse of Corollary 6 does not hold.

Example 10. Let $\mathbb{Z}$ be the ring of integers, $\mathbb{Q}$ be the field of rational numbers, $f=a_{0}+a_{1} X+\cdots+a_{n} X^{n} \in \mathbb{Z}[X]$ with $\operatorname{gcd}\left(a_{0}, a_{1}, \ldots, a_{n}\right)=1, \Delta=\{P \in$ $\operatorname{Spec}(\mathbb{Z}) \mid a_{i} \in P$ for $i=1, \ldots, n$ and $\left.a_{0} \notin P\right\}, S=\mathbb{Z} \backslash \cup_{P \in \Delta} P$, and $D=\mathbb{Z}_{S}$. Note that $f \mathbb{Q}[X] \cap \mathbb{Z}[X]=f \mathbb{Z}[X]$ and $f \mathbb{Q}[X] \cap D[X]=f D[X]$. Hence if $f$ is irreducible in $\mathbb{Q}[X]$, then $f D[X]$ is a maximal ideal by Lemma 2 , but $f \mathbb{Z}[X]$ is not a maximal ideal of $\mathbb{Z}[X]$ by Corollary 9 . (In fact, if $R$ is a ring such that $\mathbb{Z} \subseteq R \subseteq \mathbb{Q}$ and $f R[X]$ is maximal, then $D \subseteq R$.) For example, let $f=10+15 X+45 X^{3}+3 X^{4}$. Then (i) $5|10,5| 15$, and $5 \mid 45$, (ii) $5 \nmid 3$ and $5^{2} \nmid 10$, and hence $f$ is irreducible in $\mathbb{Q}[X]$ by Eisenstein's Criterion. Thus if we set $D=\mathbb{Z}_{3 \mathbb{Z}}$, then $f D[X]$ is a maximal ideal of $D[X]$, while $f \mathbb{Z}[X]$ is not a maximal ideal of $\mathbb{Z}[X]$.

Acknowledgement. The author would like to thank the referee for his/her careful reading of the manuscript and several comments. This work was supported by Basic Science Research Program through the National Research 
Foundation of Korea(NRF) funded by the Ministry of Education, Science and Technology(2010-0007069).

\section{References}

[1] D. F. Anderson and G. W. Chang, Almost splitting sets in integral domains. II, J. Pure Appl. Algebra 208 (2007), no. 1, 351-359.

[2] E. Artin and J. Tate, A note on finite ring extensions, J. Math. Soc. Japan 3 (1951), $74-77$.

[3] M. F. Atiyah and I. G. Macdonald, Introduction to Commutative Algebra, AddisonWesley, 1969.

[4] R. Gilmer, The pseudo-radical of a commutative ring, Pacific J. Math. 19 (1966), 275284.

[5] _ Multiplicative Ideal Theory, Dekker, New York, 1972.

[6] J. R. Hedstrom and E. Houston, Some remarks on star-operations, J. Pure Appl. Algebra 18 (1980), no. 1, 37-44.

[7] E. Houston and M. Zafrullah, On t-invertibility. II, Comm. Algebra 17 (1989), no. 8, 1955-1969.

[8] B. G. Kang, Prüfer v-multiplication domains and the ring $R[X]_{N_{v}}$, J. Algebra 123 (1989), no. 1, 151-170.

[9] I. Kaplansky, Commutative Rings, Revised edition. The University of Chicago Press, Chicago, Ill.-London, 1974

[10] P. May, Munshi's proof of the Nullstellensatz, Amer. Math. Monthly 110 (2003), no. 2, 133-140.

[11] M. Nagata, Local Rings, Interscience, New York, 1962.

[12] F. Zanello, When are there infinitely many irreducible elements in a principal ideal domain?, Amer. Math. Monthly 111 (2004), no. 2, 150-152.

Department of Mathematics INCHEON NATIONAL UNIVERSITY

INCHEON 406-772, KOREA

E-mail address: whan@incheon.ac.kr 